\title{
Reflective Practice and Stress: Helpful, Harmful or Uninfluential in Critical Thinking
}

\author{
Donna M. Windish, MD, MPH
}

Associate Professor of Medicine, Department of Internal Medicine, Yale University School of Medicine, New Haven, CT, USA.

J Gen Intern Med 30(9):1237-8

DOI: $10.1007 / \mathrm{s} 11606-015-3423-2$

(c) Society of General Internal Medicine 2015

$\mathrm{R}$ emember the first time you watched a master clinician work through a diagnostic dilemma or clinical unknown case and easily arrive at the correct diagnosis? That person most likely seemed calm with little stress, asked the right questions at the right time, and often reflected on the information they gleaned from the case. If the clinician described how they arrived at their diagnosis, you were probably in awe at how they solved the case using their critical thinking skills. While critical thinking is essential for effective patient care, this highly complex process is not easily taught or measured.

Critical thinking can be seen as having two components: 1) a set of information and belief generating and processing skills, and 2) the habit, based on intellectual commitment, of using those skills to guide behavior. ${ }^{1}$ Thus, while possessing medical knowledge is a necessary part of critical thinking, many factors play a role in the process, such as data gathering skills, clinical context, patient preferences, personal reactions to stress and reflective practice. Research demonstrates that some stress and thoughtful reflection are beneficial to the thinking process, ${ }^{2-4}$ yet there is no consensus on how to positively manage stress or use reflection in daily clinical care. Two articles ${ }^{5,6}$ in this issue of JGIM explore the areas of stress and reflection on critical thinking among trainees.

The study by Pottier and colleagues ${ }^{5}$ explored the role of stressors on a medical student's history taking, physical examination, and clinical reasoning. The authors performed a study of medical students who participated in two scenarios with standardized patients with familiar disease processes. Students conducted a history and physical exam and were asked to generate a diagnosis with a differential based on the clinical data obtained during the scenario. During their patient encounters, students were presented with two stressors: extrinsic and intrinsic. Extrinsic stressors were manifested by the patient's cooperativeness and mood such that the patient was either: a) aggressive with negativity and lacked confidence in the student's competence; or b) pleasant without challenges to the student. Intrinsic stressors were defined as stressful components integral to the encounter based on clinical

Published online July 15, 2015 presentation, and were exhibited by a patient with either high clinical severity (dyspnea from an acute pulmonary embolism) or low clinical severity (acute abdominal pain in a relatively healthy patient). Students were randomized to two of four clinical scenarios with a balance of high and low clinical severity in an aggressive or pleasant patient. Subjective stress and anxiety responses were assessed before and after each experience, as were completeness in physical examination, communication skills, diagnostic accuracy and differential diagnosis argumentation. The authors found that each type of stress had a different effect on a student's anxiety and clinical performance. Results indicated that extrinsic stress increased personal anxiety, but led to more accurately performing the clinical examination and increased interpersonal skills. Intrinsic stress did not change the students' anxiety, but demonstrated increases in diagnostic accuracy and differential diagnosis scores. The perceived cognitive difficulty of the task was a strong factor in most of the stress and performance variables. When the clinical scenario had high extrinsic and intrinsic stress, students no longer showed improved clinical examination or communication skills. Interestingly, the initial exposure in the first case to extrinsic and intrinsic stress did not influence the outcomes of the second case.

In their work on reflection in decision making, Monteiro and colleagues ${ }^{6}$ aimed to examine how a resident's clinical experience and self-directed decisions to reflect on their diagnosis affected the accuracy of their decisions. Using a randomized mixed design, 47 medical interns and residents were presented with 16 computerized cases where they were given a patient's primary complaint, medical history, a representative photograph, diagnostic tests and images. On their first review of the case (first pass), trainees were asked to arrive at a diagnosis as fast as they could (presumably their response in the automatic or intuitive, System 1 mode of thinking). On the second pass, residents were then randomly assigned to review either the full case again or a brief description of the case and asked to reflect on their decision and revise their diagnosis (encouraging System 2 mode analytic thinking). Residents arrived at the correct diagnosis in 58-64\% of cases with no significant differences in diagnostic accuracy among the three resident training levels. Only $8 \%$ of diagnoses were revised with minimally significant changes in diagnostic accuracy from the first pass to the second. Having access to the full case on the second pass resulted in a higher rate of diagnosis revision, but did not affect diagnostic accuracy. 
Both aforementioned studies explored factors that influence critical thinking, but limitations of these studies should be considered. First, both studies involved trainees and not practicing clinicians with more extensive medical expertise. For the medical students, their limited medical knowledge and clinical experience may have been most influential on their stress and critical thinking, as their perceived cognitive difficulty of the task itself was the greatest predictor in their performance. In the case of the medicine residents, these trainees also appeared to be limited in their medical knowledge, given their low diagnostic accuracy in the study (58$64 \%$ correct diagnoses). Thus, no amount of analytic thinking without access to outside resources may have helped improve their diagnostic thinking. Second, both studies used simulation and not patient encounters. While it is unclear how these results may be extrapolated to performance in actual clinical practice, simulation is successfully used in both teaching and evaluation in lieu of patient interactions.

While these studies have limitations, the topics of stress and reflection in critical thinking are of high interest to clinicians. Similar to what occurs in high performing athletes, the medical literature has suggested that "good" stress can positively influence performance compared to "bad" or no stress. ${ }^{2,3}$ Thus, just as athletes spend extensive amounts of time training to overcome obstacles and stress, perhaps a part of medical education should involve ways to teach students and residents how to lessen negative stress and improve positive stress. Educating trainees about personal wellness is currently being addressed by the Accreditation Council on Graduate Medical Education (ACGME), which requires each residency program to provide resident and faculty education on trainee alertness and fatigue mitigation. ${ }^{7}$ Understanding and educating about stress and its impact on clinical care may be as critical as knowledge of fatigue and its effects on personal and professional well-being. As shown in the Pottier article, having good intrinsic stress increased students' clinical reasoning, while extrinsic stress helped students focus on communication skills. These positive stress effects influenced critical thinking and the outcomes of the clinical encounter. Finding ways in the curriculum to educate medical students about the important connection between communication and critical thinking can be done successfully, as was seen in a preclinical course at Johns Hopkins University School of Medicine. ${ }^{8}$ This course found that teaching medical students the integration of communication and clinical reasoning was a viable method to improve therapeutic relationships with patients while reducing the potential for medical error and harm. Perhaps more programs should explore the effects of teaching these skills together as a way to lessen bad stress and improve good stress.

Reflective practice has been described as an essential part of professional competence. ${ }^{9}$ It is often considered to be an iterative process that occurs in more senior clinicians, especially when faced with a diagnostically challenging patient. ${ }^{10}$ While a divergence of opinion exists regarding whether or not reflective practice is useful or can be taught, educators should consider ways to best foster reflective practice early in clinical training, as it is deliberate practice and reflection that pushes us to be better clinicians and teachers.

Whether reflective practice and stress are helpful, harmful or uninfluential on critical thinking, we should all remember that our goal in medicine is to establish effective therapeutic relationships with patients while reducing diagnostic errors. Recognizing what a patient brings to an encounter with respect to their biological, cultural and psychosocial background is critical in decision making. Understanding how we each react to stressors in a clinical context and how this may negatively or positively influence our medical decision making is as important as understanding our strengths and limitations in our medical knowledge. In addition, reflection in practice, especially when taking the time to gather more information, may be critical to avoiding diagnostic errors and premature closure.

Conflict of Interest: The author declares that she does not have a conflict of interest.

Corresponding Author: Donna M. Windish, MD, MPH; Associate Professor of Medicine, Department of Internal Medicine, Yale University School of Medicine, New Haven, CT, USA (e-mail: donna.windish@yale.edu).

\section{REFERENCES}

1. Scriven M, Paul R. Critical thinking as defined by the National Council for Excellence in Critical Thinking [internet]. 1987. Available at: http://www. criticalthinking.org/pages/defining-criticalthinking/766. (last accessed April 9, 2015).

2. Chajut R, Algom D. Selective attention improves under stress: Implications for theories of social cognition. J Pers Soc Psychol. 2003;85:231-48.

3. Buchanan TW, Tranel D. Stress and emotional memory retrieval: Effects of sex and cortisol response. Neurobiol Learn Mem. 2008;89:134-41.

4. Mamede S, Schmidt H, Penaforte JC. Effects of reflective practice on the accuracy of medical diagnosis. Med Ed. 2008;42:468-75.

5. Pottier P, Hardouin JB, Dedjoie T, et al. Effect of extrinsic and intrinsic stressor on clinical skills performance in 3rd year medical student. J Gen Intern Med. 2015.

6. Monteiro SD, Sherbino J, Patel A, Mazzetti I, Norma GR, Howey E. Reflecting on diagnostic errors: taking a second look is not enough. J Gen Intern Med. 2015.

7. https://www.acgme.org/acgmeweb/Portals/0/InstitutionalRequirements_ 07012014.pdf. (last accessed April 9, 2015).

8. Windish DM, Price EG, Clever SL, Magaziner JL, Thomas PA. Teaching medical students the important connection between communication and clinical reasoning. J Gen Intern Med. 2005;20:1108-13.

9. Epstein RM, Hundert EM. Defining and assessing professional competence. JAMA. 2002;287(2):226-35.

10. Mann K, Gordon J, MacLeod A. Reflection and reflective practice in health professions education: a systematic review. Adv Health Sci Educ Theory Pract. 2009; 14(4):595-621. 